\title{
The mobile application preferences of undergraduate university students: A longitudinal study
}

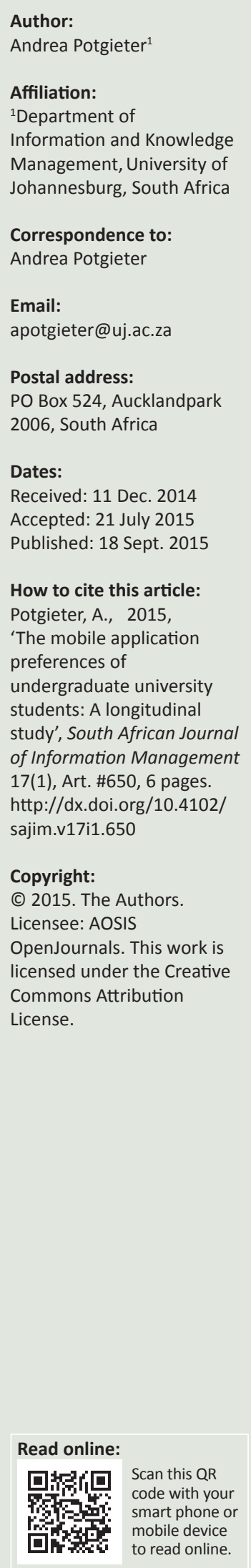

Background: Smartphones and similar mobile devices have changed the way individuals interact with technology and with each other. The app preferences of smartphone users are vitally important to those seeking to understand the motivation behind app downloads and usage.

Objective: The research problem of this article is centred on the preferences for smartphone apps by the growing market of smartphone users in South Africa. The study includes a demographic profile of the users to establish what attracts this market into downloading smartphone apps.

Methodology: The study employed a mono-method, quantitative methodological framework with an online survey as the data collection instrument. The survey was conducted amongst undergraduate university students in 2013 and repeated again in 2014.

Results: It was found that the 'young adult' demographic, of which the sample of undergraduate university students formed a part, was discerning about which apps they downloaded and that the frequency of downloads occurred less than once a month in most cases. Information and entertainment needs were amongst the top reasons users indicated as motivations for downloading apps.

Conclusion: The study's findings confirmed that the sample had definite preferences regarding which apps the users were downloading, and these preferences depended on the needs that they wished to fulfil. The study also revealed that, even though users were aware of security threats associated with downloading apps, this knowledge did not deter them from continuing to download apps. Future research recommendations also arose from the study, giving direction to prospective studies.

\section{Introduction}

Smartphones and similar mobile devices have changed the way individuals interact with technology and with one other. According to Böhmer et al. (2011:47), these devices have 'evolved from single-purpose communication devices into dynamic tools that support their users in a wide variety of tasks'. This support is mainly offered through mobile application interfaces (apps) that are designed for specific tasks and downloaded, via an app store, onto the user's smartphone. Based on trends from the literature, it is clear that changes in mobility are continuous and may branch out into many different areas, but for now apps are the main contenders (Lynch 2012; McCarthy 2014).

The effect of mobile connectivity through apps amongst students is also a global phenomenon, as $79 \%$ of young adults (ages 18-24) own smartphones, and $70 \%$ of these students are using their devices in class to stay connected (Skiba 2014). This article aims at discussing the preferences regarding mobile apps of undergraduate university students, who typically fall within the 'young adult' age group. In addition, it attempts to reveal why the respondents in the study download certain apps by highlighting the respondents' preferences for these mobile apps.

\section{Background to research problem}

According to a comprehensive Groupe Speciale Mobile Association (GSMA) study of the socioeconomic impact of the mobile industry in sub-Saharan Africa (SSA), over 6\% of the region's gross domestic product (GDP) is contributed by mobile operations, which is 'higher than any other comparable region globally' (GSMA 2013). Furthermore, according to the GSMA (2014a) country dashboard, South Africa specifically is 'a Fast Grower market in Southern Africa with four operators and 70.4 million mobile connections'.

The latest GSMA (2014b) Mobile Economy report predicts that SSA is estimated to experience the highest growth of any region with regard to the number of smartphone connections over 
the next six years. According to the report, there will be 525 million smartphone connections in the region by 2020 , and 'for the majority of users, smartphones will be the first device over which to access the Internet and to use new applications and services, as well as to explore digital content'.

It stands to reason that the app preferences of these smartphone users are vitally important to those seeking to understand the motivation behind app downloads and usage, especially when taking into account that $80.2 \%$ of South African Internet users use their app-enabled smartphones to access the Internet (Effective Measure 2014). Monica Bannan, vice president of product leadership at Nielsen, agrees that app developers should remain in the know regarding what app users expect:

As mobile consumption habits evolve, it's imperative that app developers continue to add functionality and robustness to their offerings. Although there does appear to be a limit to the number of apps people are willing to access on a monthly basis, [app users] are spending 31 percent more time than they were last year, proving that it's the content that counts. (Nielsen 2014)

Srivastava (2014), discussing the Nielsen (2014) study, argues that, due to the fact that smartphones have become an integral part of users' lives, 'the selection of specific apps has become more precautionary'. Although the number of app downloads is increasing, users have shown that they prefer to uninstall or delete any apps 'which fail to lure them within a few hours', which leads to new app developers struggling to find new and devoted users (Srivastava 2014). The research problem of this study is centred on the preferences for smartphone apps by the growing market of smartphone users in South Africa. The study includes a demographic profile of the users to establish what attracts this market into downloading smartphone apps.

To highlight these preferences, this study will present findings regarding the app proclivities of 'young adults' (a group of students in this case, the majority of whom were between the age of 18 and 25), as this group of consumers spends the most average hours on their smartphone interacting on apps when compared to other age groups. This age group attributes an average of 5.2 hours daily to smartphone use (Salesforce Marketing Cloud 2014).

The students in this sample typically fell within the 18-25 year age group, with $70 \%$ of the 2014 respondents indicating that they fall within the 18-21 year age group and $25 \%$ within the 22-25 year age group. This concentration of 'young adult' ages was also prevalent with the 2013 survey, with $73 \%$ of those respondents belonging to the 18-21 year age group and $18 \%$ within the $22-25$ year age group; see Figure 1. It has been established that this age group boasts the most active smartphone and app users, which supported the goal of this research, the search for preferences regarding mobile apps.

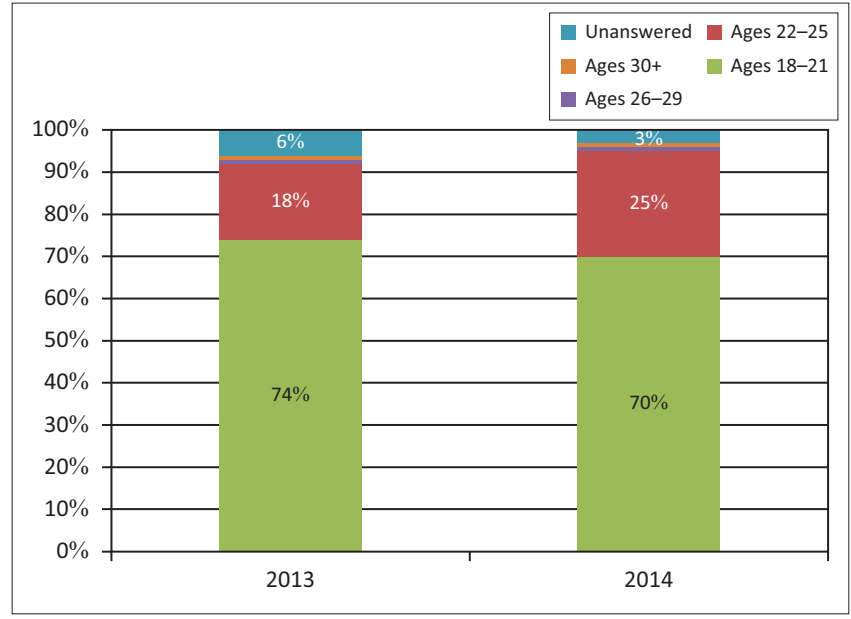

FIGURE 1: Age distribution of samples: 2013 and 2014.

\section{Research methodology}

This section constitutes the theoretical perspective of the research, discussing the overall nature of the research activity (Pickard 2013:xviii).

\section{Research design}

The research paradigm for this study was quantitative in nature, with a limited number of open-ended questions in the survey, allowing for qualitative interpretation. The philosophical paradigm in which the study was performed was one of positivism, allowing for the creation of broader generalisations based on casual relationships revealed in the data.

The research approach of this study was abductive, where the researcher aimed at generalising from the interactions between the specific and the general. The phenomenon of app choices amongst the sample group was explored in an attempt to identify themes and patterns in order to modify existing theories, or build new theories, about the subject at hand (Saunders, Lewis \& Thornhill 2012:144). The researcher employed the use of an online questionnaire to this end.

\section{Research method}

The methodological framework prevalent in this study was mono-method quantitative. Although two open-ended questions were present in the survey, the terms that arose from those questions were analysed based on the frequency of each term appearing. The data from these open-ended questions were inducted in a quantitative format, as was the case with the typical Likert-scale questions collected throughout the rest of the questionnaire.

\section{Data collection instrument}

The online survey used in the 2014 study was a marginally modified version of the survey implemented by Mashiane and Potgieter in 2013 (Mashiane \& Potgieter 2014) for the purpose of comparing results longitudinally. In both 2013 
and 2014, the survey was deployed to the sample group through the use of the online university student portal, ULink. This portal offers a survey facility which deploys a survey directly to the relevant users - in this case, undergraduate university students.

The questionnaire consisted of 28 questions, four of which were open-ended questions where respondents could type in the relevant answer. The remaining 24 questions were delivered in a multiple-choice style, giving the respondents the option of choosing the appropriate answer, or a neutral answer ('I don't know' or 'Other') where applicable.

The findings of the 2013 Mashiane and Potgieter (2014) survey were presented at the Pan-Pacific Conference XXXI in Japan in June 2014. The findings of the 2014 survey were presented at the Annual Information and Knowledge Management Conference in South Africa in November 2014. This article will compare and discuss the findings of these two data collection instances.

\section{Survey samples}

The sampling technique applied in both data collection instances was one of non-probability convenience sampling, which is appropriate in the quantitative nature of the study's research method (Kumar 2014:242). Saunders et al. (2012:176) notes that samples that are selected based on convenience are characteristically easier to access, because these samples are typically familiar to the researcher. Saunders et al. (2012:291) also state that, in many instances, samples chosen for convenience seemingly 'meet purposive sample selection criteria that is relevant to the research aim'. Since this study focused on the tendencies of young adults and their preferences regarding app downloads, the sample was both convenient whilst also meeting the purposive criteria of falling within the 'young adult' range.

Using the University of Johannesburg's (UJ) online student portal ULink, the survey was made available to all undergraduate Information Management students at the UJ in June 2013 and again in September 2014. The survey was developed by taking into consideration global trends in mobile app usage surveys. Contemporary surveys were used as guidelines in this endeavour.

In 2013, the survey was made available to 1161 students, the total number of students registered for the subject Information Management on an undergraduate level at the time of the study. A response rate of $62 \%$ was achieved with a total of 717 responses successfully captured.

During the 2014 data collection period, September to November, the survey was posted to all undergraduate Information Management students at the UJ. In September 2014, the survey was made available to 1256 undergraduate students, and by 05 November 2014, 522 responses had been captured, amounting to a response rate of $42 \%$.
In both instances, an online survey was selected, since these students access their student portal on a regular basis. The suitability of being able to participate in the survey by using a portal they are familiar with was considered to be an advantage.

\section{Findings}

Survey respondents were asked whether they owned an Internet-enabled smartphone. There was a slight increase in the number of respondents as $79 \%$ of respondents in 2013 indicated that they owned a smartphone, compared to $84 \%$ in 2014 . In both instances, this percentage was higher than the smartphone penetration reported for urban dwellers in South Africa, which is 62\% (On Device Research 2014).

Subsequently, respondents were asked whether they downloaded apps to their smartphones. This number increased to $77 \%$ in 2014, and the increase was to be expected, since apps have been described as 'the single most significant tool driving the mobile economy in South Africa' (World Wide Worx 2013).

In 2013,44\% of respondents indicated that they owned a smartphone with a BlackBerry operating system (OS), and only $13 \%$ of respondents owned an Android enabled smartphone. In 2014, BlackBerry was no longer the leader amongst the sample, with only $25 \%$ of respondents indicating that they owned a Blackberry device and 37\% representing Android; see Figure 2. The loss of market share by BlackBerry had been predicted by Effective Measure (2014); it had also been predicted that the market share would be lost to Samsung (an Android device) and - at least from an OS point of view - is seemingly confirmed in this instance.

When respondents were asked when they searched for an app, the motivation for 2013 and 2014 searches were relatively similar; see Figure 3. A notable difference between the two instances was the most popular reason respondents had pointed out as motivation for searching for an app. In $2013,48 \%$ of respondents indicated that they searched for an app when they 'need information on a brand, its product or its services', whereas the most popular reason in 2014 for

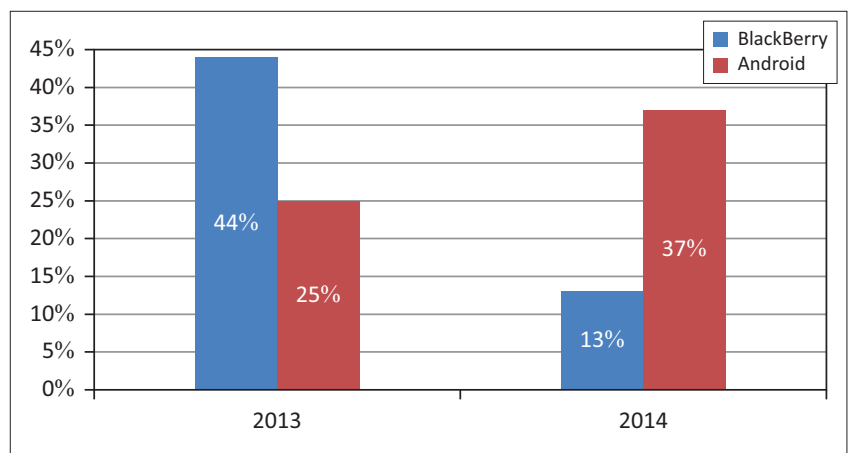

FIGURE 2: Growth of Android adoption from 2013-2014. 


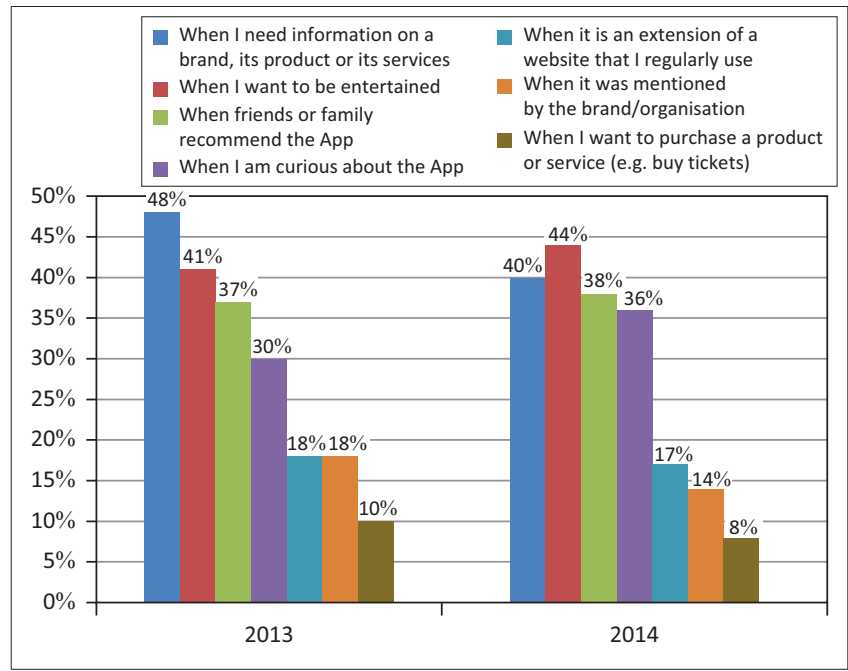

FIGURE 3: Motivation for searching for an app, 2013 vs. 2014.

searching for an app was that respondents 'wanted to be entertained'.

In both 2013 and 2014, the remaining reasons noted for searching for an app were indicated as follows, in descending order of preference:

- When friends or family recommend the app.

- When I am curious about the app.

- When it is an extension of a website that I regularly use.

- When it was mentioned by the brand and/or organisation.

- When I want to purchase a product or service (e.g. buy tickets).

What these findings imply, is that an information need and a need to be entertained are evidently equally important to this sample. The reasons for searching for an app as listed above, are typically less important, but also indicate a hierarchy in the samples' motivations for searching for apps to download. Based on this finding, one could infer that a respondent will most probably search for an app when he or she is bored, or needs information about a product. They will be less likely to do so if an app is an extension of a website that they typically use, or if they want to purchase a product or service.

Respondents were also asked what they considered before downloading an app that they had searched for. Respondents indicated, in both 2013 and 2014, that the price of the app was the most important feature. It must, however, be noted that Pengnate and Delen (2014:7), when controlling for confusing factors such as price, found a strong relationship between 'emotions and the number of an app downloaded', and state that this relationship can hypothetically be described 'by the affect-as-information model and theory of reasoned action'. Therefore, even though the respondents in this sample indicated that price plays a very important role in their selection of an app, they may not be aware of the underlying emotional triggers affecting their choices.

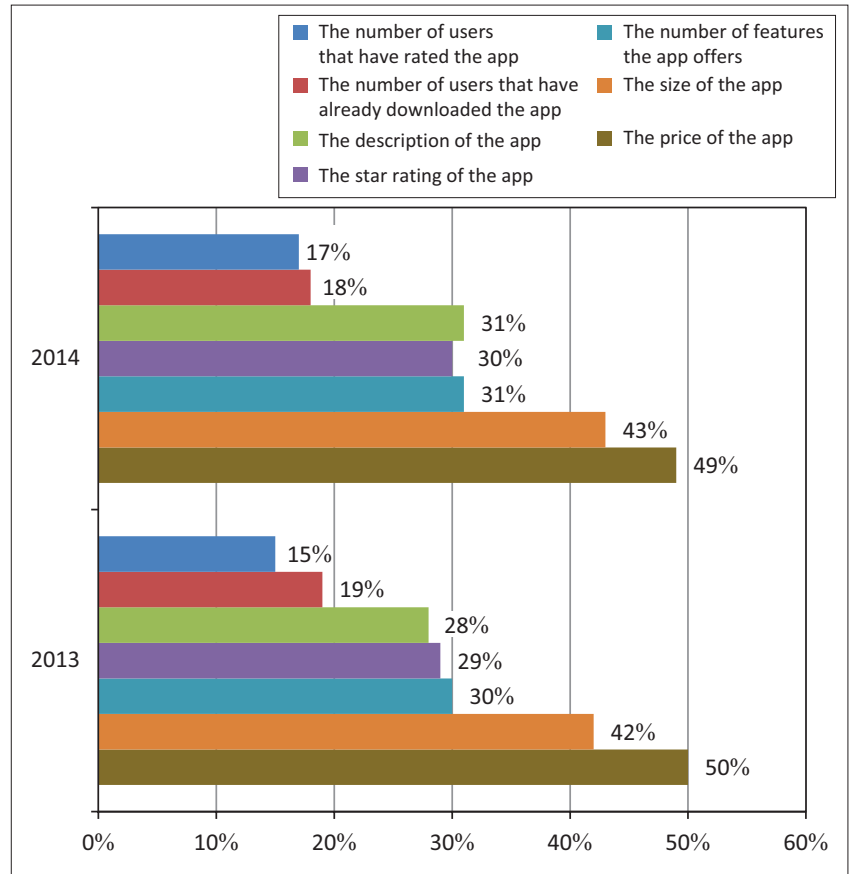

FIGURE 4: Considerations before downloading an app, 2013 vs. 2014.

An important secondary consideration was the size of the actual app, possibly implying that device memory is a consideration for this demographic as it influences device performance (Huang et al. 2010). Khalid et al. (2015:74) confirm this notion by remarking that app users notably complained about 'resource heavy' apps; these apps consumed too much battery life or memory on mobile devices, which caused frustration with regard to performance.

Equally important to one another, but less important overall, was the number of features the app had to offer, the star rating of the app on the app store platform, and the app's description. Pagano and Maalej (2013:132) note a bias in user rating of apps, as users may consider feedback which gives an app a low rating as less helpful than feedback giving an app a higher rating. It becomes evident that feedback which gives an app a better rating is considered more helpful by users, who then rate these positive reviews as more helpful. Taking the partiality of the app rating system into account, it is satisfactory to note that the least consequential of considerations that the respondents in this study took into account before downloading an app were the number of users that have rated the app, and the number of users that have already downloaded the app. This was the case with both the 2013 and the 2014 respondents; see Figure 4.

The issue of security risks was raised with respondents. In $2013,53 \%$ of the respondents indicated a concern regarding security risks when downloading apps, and in 2014, 51\% of the respondents indicated a similar concern. The risks associated with downloading mobile apps are real as a MetaIntell study found that $92 \%$ of the top 500 downloadable Android apps carry security or privacy risks (Business Wire 2014). Although it is evident that these respondents are 
aware and concerned about the security risks associated with downloading apps to their phones, it did not deter the majority of respondents from downloading apps regardless of these risks.

Respondents were asked to indicate how many apps they download per month. In 2013, 33\% of respondents noted that they do not download apps every month. This percentage remained similar in 2014, when $34 \%$ of respondents indicated that they do not download apps on a monthly basis. In 2013 and 2014 respectively, 25\% and 22\% of respondents indicated that they downloaded at least one app per month; $17 \%$ and $16 \%$ agreed that they downloaded two to five apps per month. Very few of the respondents downloaded more than five apps per month, with only $5 \%$ of respondents in 2013 and 6\% in 2014 indicating that they downloaded apps at this rate.

This finding supported the exploration of the research problem of this study, as it was evident that this demographic, although actively using their smartphones according to global standards, were very discerning when downloading apps. The majority of respondents, in both instances, indicated that they do not download apps more than once a month. This suggested that specific criteria were taken into account by these users when they decided to download an app.

The app that most respondents had downloaded at the time of the survey changed from 2013-2014. In 2013, Facebook was a clear favourite with $75 \%$ of respondents indicating that they had this app on their smartphone, whereas in 2014, WhatsApp had been installed by $73 \%$ of respondents - the highest instance compared to other apps. Unfortunately, many smartphones have Facebook pre-installed at purchase; therefore, it is not a clear indication of whether the respondents had downloaded the app out of their own volition. It does however confirm that this app had not been uninstalled once the device had been personalised by the respondents. Further study of the issue could possibly explore which pre-installed apps remain installed and used on users' phones after purchase.

The fact that WhatsApp was to be found on the majority of the 2014 sample's smartphones was a testament to this app's popularity, as WhatsApp is not typically pre-installed at purchase. The attractiveness of WhatsApp was further confirmed when respondents selected this app as 'the most useful app they had ever downloaded'; $18 \%$ of respondents in 2013 and 22\% in 2014 sided with WhatsApp in their answers to this open-ended question. The remaining responses were divided, in less frequent instances, between apps such as Facebook, News24 and Opera Mini.

When the question of whether respondents would consider downloading paid-for apps was asked, the response was mostly negative from the $2013(42 \%)$ and the $2014(46 \%)$ sample groups. Considering that $32 \%$ of respondents in 2013 and $25 \%$ of respondents in 2014 'did not have a monthly income', and 43\% of respondents in 2013 and 28\% in 2014 had a monthly income of less that R1000, it was to be expected that paying for apps would not be a priority.

Even though the general attitude seemed to be not to pay for apps, 22\% of respondents in 2013 and 17\% in 2014 indicated that they had at least one paid-for app installed on their smartphone. The main reasons indicated by respondents that led them to consider paying for an app were 'if the paid-for app appeared to be of a higher quality than a free app that offers the same function' (33\% in 2013 and 30\% in 2014), or 'if a paid-for app fulfilled a specific need that the respondent considered vital' (28\% in both 2013 and 2014).

In 2013, 28\% of respondents also indicated that the most they had ever paid for an app had been 'less than R10', whereas $23 \%$ indicated the same in 2014. A smaller percentage of respondents in $2013(18 \%)$ and 2014 (19\%) confirmed that the most they had ever paid for an app had been 'between R11 and R50'. Even fewer respondents indicated that they had ever paid 'between R51 and R100' for an app, with merely 5\% of respondents in 2013 and 4\% in 2014. Only 1\% of respondents in both 2013 and 2014 indicated that they had ever paid 'more than R100' for an app. This finding can also be linked to the low income bracket of the sample in both 2013 and 2014.

\section{Conclusion and recommendations}

This longitudinal study set out to establish the preferences regarding downloading apps of undergraduate university students. Following the analysis of data from two samples, one surveyed in 2013 and the other in 2014, it was found that these groups of 'young adults' were discerning about which apps they download, as the frequency of downloads was relatively low. It was found that less than one app per month was being downloaded by the respondents.

The main motivation for downloading apps was found to be either a need for specific information (in 2013), or a need to be entertained (in 2014). It was also found that the price and size of an app were important considerations when respondents considered downloading an app. The recurring theme of app price was evident, as respondents either had a very small monthly income or no income at all, making the downloading of paid-for apps a non-probability.

On the rare occasions where respondents did download paid-for apps, the motivation had been a perceived higher quality of the paid-for app compared to its free counterparts. In addition to a perceived higher quality of paid-for apps, respondents also indicated that they would choose to purchase one if they considered the paid-for app to be vital to fulfil a specific need. When respondents committed to paying for an app, it was found that most chose an app that cost less than R10 to download.

WhatsApp was found to be a very popular app downloaded by the sample groups. It is suggested that a follow-up study 
be conducted to establish whether 'popular' apps are apps that had been pre-installed before purchase, as was the case with Facebook. Since respondents indicated that the need to access information and to be entertained were important considerations when downloading apps, it stands to reason that these two popular apps, WhatsApp and Facebook, most likely fulfilled these needs.

The study also showed that app users were aware of the security risks associated with downloading apps to their smartphones, but that these risks were not deterring the download of apps. This is an interesting finding, as it could indicate that these respondents did not regard a threat to their information privacy as a cause for concern; further investigation is needed to explore the issue of security risks in the downloading of apps.

Although some insight was given into the preferences of this demographic with regard to app use, this area of study still poses many unanswered questions, the disregard of risk regarding security when downloading apps being one example. As the use of apps increases, as it has in recent times, these questions need to be explored in order for app developers to create useful products, and also for researchers to gain insight into the interaction of users with their smartphones.

It is further suggested, for future research, to not exclude older generations from research in smartphone and app usage research simply because they have been slower in adopting the technology. For the purpose of this study, the sample explored 'young adults' as this demographic was found to spend the most time interacting using their smartphones. According to Deloitte (2014), however, smartphone penetration by individuals over 55 is on the rise and by 2020 the current gap between this age group and younger demographics will be negligible. A study comparing the differences in app usage amongst all age groups, in South Africa specifically, will offer crucial insight.

\section{Acknowledgements Competing interests}

The authors declare that they have no financial or personal relationships which may have inappropriately influenced them in writing this article.

\section{References}

Böhmer, M., Hecht, B., Schöning, J., Krüger, A. \& Bauer, B., 2011, 'Falling asleep with Angry Birds, Facebook and Kindle - A large scale study on mobile application usage', Proceedings of the 13th International Conference on Human Computer Interaction with Mobile Devices and Services, Stockholm, Sweden, August 30 September 2, 2011, pp. 47-56.

Business Wire, 2014, Metalntell identifies enterprise security risks, privacy risks and data leakage in $92 \%$ of top 500 android mobile applications, viewed 22 January 2014, from http://www.businesswire.com/news/home/20140122006295/en/ Metalntell-Identifies-Enterprise-Security-Risks-Privacy-Risks\#.VIcDp9KUcrV

Deloitte, 2014, The smartphone generation gap: Over-55? There's no app for that, viewed 26 October 2014, from http://www2.deloitte.com/content/dam/ Deloitte/global/Documents/Technology-Media-Telecommunications/gx-tmt2014prediction-smartphone.pdf

Effective Measure, 2014, South African mobile report: A survey of desktop user's attitudes and uses of mobile phones, viewed 26 October 2014, from http://www. effectivemeasure.com/south-african-mobile-report-march-2014

GSMA, 2013, New GSMA report shows sub-Saharan Africa lea ds the world in mobile growth and impact, media release, viewed 11 November 2013, from http://www. gsma.com/newsroom/sub-saharan-africa-leads-world/

GSMA, 2014a, Data » Markets » Africa » South Africa, viewed 28 October 2014, from https://gsmaintelligence.com/markets/3788/dashboard/

GSMA, 2014b, The mobile economy: Sub-Saharan Africa 2014, viewed 28 October 2014, from http://ssa.gsmamobileeconomy.com/

Huang, J., Xu, Q., Tiwana, B., Mao, Z.M., Zhang, M. \& Bahl, P., 2010, 'Anatomizing application performance differences on smartphones', Proceedings of the 8th international conference on mobile systems, applications, and services, San Francisco, USA, June 15-18, 2010, pp. 165-178.

Khalid, H., Shihab, E., Nagappan, M. \& Hassan, A.E., 2015, 'What do mobile app users complain about?', Software, IEEE 32(3), 70-77. http://dx.doi.org/10.1109/MS.2014.50

Kumar, R., 2014, Research methodology: A step by step guide for beginners, 4th edn., Sage Publishing Ltd., London.

Lynch, W., 2012, How apps are taking over computing and content, Web Log Post, viewed 20 November 2012, from http://www.realmdigital.co.za/post/how-appsare-taking-over-computing-and-content/

Mashiane, S. \& Potgieter, A., 2014, 'Enterprise apps: What do consumers really think?', Proceedings of the Pan-Pacific Conference XXXI, Osaka, Japan, June 2-5, 2014, pp. 70-72.

McCarthy, N., 2014, 'Mobile app usage by the numbers [infographic]', viewed 29 October 2014, from http://www.forbes.com/sites/niallmccarthy/2014/10/29/ mobile-app-usage-by-the-numbers-infographic/

Nielsen, 2014, Smartphones: So many apps, so much time, viewed 01 July 2014, from http://www.nielsen.com/us/en/insights/news/2014/smartphones-so-manyapps--so-much-time.html

On Device Research, 2014, Impact of the mobile Internet in Africa vs UK, viewed 22 October 2014, from http://www.slideshare.net/OnDevice/impact-of-the-mobileinternet-in-african-lives? redirected_from=save_on_embed

Pagano, D. \& Maalej, W., 2013, 'User feedback in the AppStore: An empirical study', Proceedings of the IEEE 21st International Requirements Engineering Conference (RE), Rio de Janeiro, Brazil, July 15-19, 2013, pp. 125-134.

Pengnate, S.F. \& Delen, D., 2014, 'Evaluating emotions in mobile application descriptions: Sentiment analysis approach', Proceedings of the 20th Americas Conference on Information Systems, Savannah, USA, August 7-9, 2014.

Pickard, A.J., 2013, Research methods in information, Facet Publishing, London.

Salesforce Marketing Cloud, 2014, 2014 mobile behavior report, viewed 26 October 2014, http://www.exacttarget.com/2014-mobile-behavior-report

Saunders, M., Lewis, P. \& Thornhill, A., 2012, Research methods for business students, Pearson, Essex.

Skiba, D.J., 2014, 'The connected age: Mobile apps and consumer engagement', Nursing Education Perspectives 35(3), 199-201. http://dx.doi.org/10.5480/15365026-35.3.199

Srivastava, B., 2014, Mobile app usage to rise, but new apps struggle to find market! viewed 17 July 2014, from http://www.dazeinfo.com/2014/07/17/mobile-appusage-us-2013-catagory-interest-age-group-study/

World Wide Worx, 2013, The time of the app, viewed 28 November 2013, from http:// www.worldwideworx.com/mobileinternet2014/ 